\title{
Effects of Electric Stimulation with Static Stretching on Hamstrings Flexibility
}

\author{
Won-Min Song', Hye-Jeong Seo ${ }^{2}$, Won-Seob Shin ${ }^{3}$ \\ 'Department of Physical Therapy, College of Health and Medical Science, Daejeon University; ${ }^{2}$ Department of Physical Therapy, Graduate School of \\ Daejeon University; ${ }^{3}$ Department of Physical Therapy, College of Health and Medical Science, Daejeon University, Daejeon, Korea
}

Purpose: Flexibility has been considered one of the most important goals in rehabilitation. This study aimed to investigate the effect of transcutaneous electrical nerve stimulation (TENS) with the static stretching technique on the flexibility of hamstrings.

Methods: Twenty-four subjects (15 men, 9 women) with limited hamstrings flexibility received three different intervention sessions in random order. The treatment sessions included static stretching (SS), static stretching with motor-level TENS (SS with motor TENS) and sensory-level TENS (SS with sensory TENS). All sessions of SS were performed in the straight-leg raise position for 30 seconds followed by rest for 15 seconds, in repetitions for 10 minutes using a belt. The TENS groups underwent TENS stimulation (40 $\mu \mathrm{s}, 100 \mathrm{~Hz})$ during the stretching for 10 minutes. Outcome measures were evaluated according to active knee extension (AKE) and recorded before the session and at 0, 3, 6, 9, and 15 minutes after the session.

Results: There was significant improvement in hamstrings flexibility within all groups $(p<0.05)$. SS with TENS (both sensory and motor) maintained significant increases in knee extension range of motion until 15 minutes post-treatment. In contrast, the SS-only group maintained significantly increased hamstrings flexibility only until 6 minutes post-treatment $(p<0.05)$.

Conclusion: Improvement in hamstrings flexibility with SS with TENS was maintained longer than with SS-only intervention. Electrical stimulation with stretching may be more effective than SS alone for increased duration of maintained hamstrings flexibility.

Keywords: Hamstrings flexibility, Static stretching, Electrical stimulation

\section{INTRODUCTION}

Flexibility has been defined as the ability of a muscle to lengthen and to allow one joint (or more than one joint in a series) to move through a range of motion (ROM) without limitation or pain., Flexibility of muscles is an essential component for injury-prevention and rehabilitation. ${ }^{3}$ Muscle tightness is caused by a decrease in the ability of the muscle to deform, resulting in decreased ROM of the joint on which it acts. ${ }^{4}$ Plans to maintain the flexibility of muscles are of concern to physical therapists, physical educators, and rehabilitation specialists. ${ }^{1,2}$

The length of the hamstrings muscles is considered to play an important role in both the efficiency of basic human movements, such

Received May 29, 2015 Revised Jun 15, 2015

Accepted Jun 18, 2015

Corresponding author Won-Seob Shin

E-mail Shinws@dju.kr as walking and running, and their effectiveness. ${ }^{5}$ Hamstrings shortening may affect the fundamental features as well as walking-posture changes associated with various musculoskeletal disorders related to the lumbar spine, such as low back pain. ${ }^{5,6}$ Therefore, it is necessary to increase the flexibility of the hamstrings by stretching for people with shortened hamstrings.

Clinically, flexibility has been considered one of the most important goals in rehabilitation, and stretching techniques are mainly used in exercises for preparing and finishing athletes for competition, and for the general public., ${ }^{7.8}$ To increase hamstrings flexibility, static stretching (SS), ballistic stretching, proprioceptive neuromuscular facilitation (PNF), and dynamic stretching have mainly been used in previous stretching interventions. ${ }^{9,10}$ Of those, SS has been

Copylight (C2015 The Korea Society of Physical Therapy

This is an Open Access article distribute under the terms of the Creative Commons Attribution Non-commercial License (Http:// creativecommons.org/license/by-nc/3.o.) which permits unrestricted non-commercial use, distribution, and reproduction in any medium, provided the original work is properly cited. 
the most widely used because it is easy to apply and results in less soft-tissue damage while maintaining maximum muscle length. ${ }^{1,11}$ Many previous studies found that SS was a very effective technique. ${ }^{1,11-13}$

When stretching exercises are done for muscles with reduced flexibility, pain occurs in the soft tissues, which is defined as 'stretch pain'. ${ }^{14}$ Commonly, transcutaneous electrical nerve stimulation (TENS), hot packs, and ice are used for the purpose of reducing pain. ${ }^{15}$ Conventional TENS changes the pain sensation using a counter-stimulation effect based on the gate-control theory. ${ }^{16}$ TENS is widely used clinically, mainly for the purpose of pain control, because it is a non-invasive, nonpharmacological intervention that is easy to use. ${ }^{14,17}$ The duration and effect of hamstrings flexibility gains after SS with TENS has undergone limited research. The current study evaluates the effect of SS with TENS and compares it to normal SS in patients with limited hamstrings flexibility.

\section{METHODS}

\section{Subjects}

The subjects were 24 individuals (15 men, 9 women) with limited hamstrings flexibility. The average age of subjects was $23.4 \pm 1.6$ years, the average height was $169.0 \pm 7.3 \mathrm{~cm}$ and the average weight was $61.9 \pm 8.1 \mathrm{~kg}$. All subjects had hamstrings tightness, defined as a loss of $30^{\circ}$ or more from full extension with the hip at $90^{\circ} .^{7}$ All subjects had no history of orthopedic or neurologic disorders in the hips, knees, or lower back for the previous 3 months. The subjects were informed about the purpose of the present study and its procedures, and written informed consent was obtained from all subjects.

\section{Instruments and measurement}

To evaluate the effect of stretching, active knee extension (AKE), passive knee extension (PKE), sit-and-reach, and straight-leg raise (SLR) have traditionally been used. ${ }^{7}$ Clinically, the AKE test is often recommended because it eliminates pelvic rotation and measured hamstrings length selectively than others. In the supine position, the ipsilateral hip is positioned at $90^{\circ}$ of flexion and the subject fully extends the knee joint. The AKE test has a high test-retest reliability $(0.99))^{18}$

The hamstrings flexibility measurement procedures in this study were as follows: in the supine position, the subject placed his or her dominant leg on a fixed metal experimental frame that maintained the $90^{\circ}$ flexion position, and a towel was placed between the T12 and L5 vertebral levels to minimize the effect of other movements on the results. The non-dominant leg was fixed by a strap in the middle of the thigh. ${ }^{719}$ The angle of knee extension was measured with an electronic inclinometer (Dualer IQ the smarter inclinometer, JTECH medical, Salt lake, USA), which is a reliable measurement with high intraclass correlation coefficients (ICC-3, k) of $>0.95 .{ }^{20}$ AKE angle (degree) and increased flexibility percentage from the initial angle was compared of each time.

\section{Intervention}

The subjects, who all had limited hamstrings flexibility, received 3 different intervention sessions in random order: SS alone, SS with motor TENS, and SS with sensory TENS. AKE was used to evaluate the flexibility of the hamstrings, with measurements at the beginning of the study and 0,3, 6, 9, and 15 minutes after each stretching program. ${ }^{7}$ Between each treatment, a break was taken for at least 24 hours to minimize carry-over effects. Each stretching intervention was performed for 30 seconds with rest intervals of 15 seconds, and repetitions were performed for 10 minutes. ${ }^{19}$

\section{1) Static stretching (SS)}

In the supine position, each subject maintained a full knee-extended position with ankle joint neutrality $\left(90^{\circ}\right)$, and stretched the hamstrings muscle passively to the point of a "strong, but tolerable stretch" until feeling no pain, using a belt. ${ }^{21}$ The participants performed the stretches for 30 seconds, rested for 15 seconds, and repeated the cycle for 10 minutes.

\section{2) Static stretching with TENS}

During SS, two electrodes were applied in parallel on the upper and lower hamstrings muscles for 10 minutes $(100 \mathrm{~Hz}, 40 \mu \mathrm{s}){ }^{22}$ Sensorylevel TENS was set at an intensity that felt most comfortable. Motorlevel TENS was applied to a level of discomfort, at least until visible contractions were induced, starting with $1 \mathrm{~mA}^{23}$

\section{Statistical analyses}

All statistical analyses were conducted using SPSS version 18.0 (SPSS Inc., Chicago, IL, USA). The general characteristics of the subjects were reported as the mean and standard deviation of the 
descriptive statistics. To determine the normal distribution of the measured values, the Kolmogorov-Smirnov test was used. One-way repeated ANOVA was used to compare between the three interventions. The three stretching interventions' time measurements were analyzed by one-way ANOVA. A post-hoc Tukey test was used for the statistical significance of results. Statistical significance was defined as a p-value of $<0.05$.

\section{RESULTS}

\section{Comparison AKE in within-group}

There were significant changes in the measurement points regardless of group $(\mathrm{p}<0.05)$, but there were no differences in hamstrings flexibility of the three groups before the intervention $(\mathrm{p}<0.05)$. Measurements at 3,6, and 9 minutes after the intervention per the angle of flexibility were different from before SS intervention $(\mathrm{p}<0.05)$. Also, in SS with sensory- and motor-level TENS, measurements of flexibility of the angle had significant differences at 3 , 6,9 , and 15 minutes after the intervention, compared to before the intervention $(\mathrm{p}<0.05)$ (Table 1).

\section{Comparison AKE in between-groups}

There were no significant differences before the intervention or 3 minutes after the intervention among the three groups. After 6, 9, and 15 minutes, hamstrings flexibility was different in the three stretching groups $(\mathrm{p}<0.05)$. After 6 and 9 minutes, each group (SS alone compared with SS with motor TENS, and SS with sensory TENS compared to SS with motor TENS) had significant differences in hamstrings flexibility $(\mathrm{p}<0.05)$. After 15 minutes, only the SS with motor TENS group had significant differences in hamstrings flexibility $(\mathrm{p}<0.05)($ Table 1$)$.

\section{DISCUSSION}

This study aimed to evaluate the effects of three stretching interventions and the subsequent duration of increased flexibility over time, targeting young men and women with limited hamstrings flexibility. TENS applied intensity of this study was different depending on individual perception. There was only one study that combined stretching exercise and TENS without distinction of TENS intensity level. ${ }^{22}$ However, this study was divided into sensory-level TENS and motor-level TENS to evaluate the result. Approximately, sensory level TENS intensity was 16-38 mA and motor level TENS intensity was 55-65 mA.

In a previous study, the SS technique helped to increase ROM, and SS combined with TENS or cold was more effective than SS alone. ${ }^{1,24}$ This study also showed a significant increase in hamstrings flexibility with SS alone and SS applied with TENS, compared to before the intervention. However, the SS combined with TENS group showed a greater incremental increase than did the SS group. Of these, the SS with motor TENS group was the highest (20.74\%) when compared to the SS with sensory group (18.80\%) and the normal-SS group (16.85\%).

To induce the contraction of muscle to increase flexibility, the techniques of hold-relax, eccentric contractions, etc. have been reported to influence flexibility through muscle-neurological mechanisms. ${ }^{8,10}$ Based on the proprioceptive neuromuscular facilitation (PNF) technique, the hold-relax technique induces relaxation after a maximum isometric contraction of the muscles that need increased flexibility. ${ }^{3}$ Eccentric contraction is a technique that applies dynamic extension of the agonist and contraction of the antagonist. In this way, metabolism is increased and there is improved flexibility of muscles via the local temperature increase in the deep muscles,

Table 1. A comparison of AKE angle after static stretching interventions

(degree $\left(^{\circ}\right)$ )

\begin{tabular}{|c|c|c|c|c|c|c|c|c|}
\hline & SS $^{A}$ & Increment (\%) & $S S+$ STENS ${ }^{B}$ & Increment (\%) & $\mathrm{SS}+\mathrm{mTENS} \mathrm{C}$ & Increment (\%) & $\mathrm{F}$ & Post-hoc \\
\hline Baseline & $48.96 \pm 7.56$ & & $49.42 \pm 6.52$ & & $50.04 \pm 7.09$ & & 0.74 & - \\
\hline Post & $57.21 \pm 6.57^{+}$ & 16.85 & $58.71 \pm 5.20^{+}$ & 18.80 & $60.42 \pm 6.61^{+}$ & 20.74 & 3.12 & - \\
\hline After $3 \mathrm{~m}$ & $54.79 \pm 6.91^{+}$ & 11.91 & $55.92 \pm 5.34^{+}$ & 13.15 & $57.54 \pm 5.86^{+}$ & 14.99 & 2.81 & - \\
\hline After $6 \mathrm{~m}$ & $53.04 \pm 6.46^{+}$ & 8.33 & $54.63 \pm 5.00^{+}$ & 10.54 & $56.75 \pm 6.02^{+}$ & 13.41 & $9.09^{\neq}$ & $A, B<C$ \\
\hline After $9 \mathrm{~m}$ & $51.83 \pm 6.26^{+}$ & 5.86 & $53.42 \pm 5.29^{+}$ & 8.09 & $55.67 \pm 5.42^{+}$ & 11.25 & $10.08^{+}$ & $A, B<C$ \\
\hline After $15 \mathrm{~m}$ & $50.21 \pm 7.84$ & 2.55 & $52.29 \pm 5.99^{+}$ & 5.81 & $54.42 \pm 5.52^{+}$ & 8.75 & $6.33^{+}$ & $A, B<C$ \\
\hline $\mathrm{F}$ & $41.51^{*}$ & & $50.04^{*}$ & & $48.46^{*}$ & & & \\
\hline
\end{tabular}

*significant different within group $(p<0.05)$; significant different compared from Baseline $(p<0.05)$; ${ }^{*}$ significant different between intervention group ( $\left.<<0.05\right)$. SS, static stretching; SS+sTENS, static stretching with sensory level TENS; SS+mTENS, static stretching with motor level TENS. 
which reduces the viscoelasticity of the muscle-tendon unit. ${ }^{7}$ SS with motor-level TENS, used in this study, is similar to the muscleneurological mechanism of the above techniques when the TENS is applied at a level that causes visible contractions of the hamstrings.

Compared to the holding time between the three groups, the normal-SS group maintained a growth rate of 5.81\% to 9 minutes, and the SS with sensory TENS and the SS with motor TENS groups growth rates were $8.57 \%$ and $5.81 \%$, respectively, after 15 minutes. Previous studies have described the use of conventional TENS, originally based on the gate-control theory of pain, which suggested that counter-stimulation of the nervous system could modify the perception of pain. ${ }^{16,25}$ This is a mechanism for adjusting the level of pain in the spinal cord. The pain-transmission path from the outside of the body through the skin occurs via the primary afferent nerves, passing to the brain through the dorsal column or the anterolateral fasciculus, at which point the individual becomes aware of the pain. Stimulation of large-diameter afferent fibers inhibits second-order neurons in the dorsal horn and blocks pain carried by small-diameter fibers from reaching the top. ${ }^{26}$ Compared with normal SS, the SS with TENS technique may have influenced the effect, similar to the gate-control theory, adjusted to reduce pain when stretching the hamstrings. The results of the present study show that SS with TENS is more effective in increasing flexibility compared to SS alone, and the groups applying TENS maintained increased flexibility for up to 15 minutes. This result may be due to the muscleneurological mechanisms of muscle contraction and the gate-control theory of TENS acting in combination.

The effects of muscle stretching are known to include reduced muscular soreness, enhanced physical fitness, reduced risk of joint sprain or muscle strain, and promotion of the development of body awareness. ${ }^{27}$ However, stretching pain, defined as pain associated with stretch stimulations in muscles, accompanies stretching exercises. Therefore, stretch-pain control is necessary in order to increase flexibility. By stimulating a peripheral nerve, SS with TENS may help prevent muscle fatigue and alleviate muscle spasms, and it could be utilized clinically.

The limitations of this study were that hamstrings flexibility was measured only with the AKE test, and the TENS setting of $40 \mu \mathrm{s}$, $100 \mathrm{~Hz}$ was fixed, so that different frequencies and pulse wave periods were not known. In future studies, the appropriate frequency and pulse for TENS, as well as various measurement techniques, will need to be researched.

\section{REFERENCES}

1. Nelson RT, Bandy WD. Eccentric training and static stretching improve hamstring flexibility of high school males. J Athl Train. 2004;39(3):254-8.

2. Lee DK, Kim YN. The effects of proprioceptive neuromuscular facilitation lower extremity pattern on muscular strength and flexibility in an aquatic environment. J Kor Soc Phys Ther. 2013;25(2):49-55.

3. Spernoga SG, Uhl TL, Arnold BL, et al. Duration of maintained hamstring flexibility after a one-time, modified hold-relax stretching protocol. J Athl Train. 2001;36(1):44-8.

4. Akinpelu AO, Bakare U, Adegoke BA. Influence of age on hamstring tightness in apparently healthy Nigerians. Journal of the Nigeria Society of Physiotherapy. 2009;15(2):35-41.

5. Ayala F, de Baranda PS, Croix MDS, et al. Comparison of active stretching technique in males with normal and limited hamstring flexibility. Phys Ther Sport. 2013;14(2):98-104.

6. Park YN, Bae YS, Comparison of muscle performance of the lumbar region and head alignment according to the length of sitting time. J Kor Soc Phys Ther. 2013;25(6):386-92.

7. Moon AY, Jang HJ, Jang HJ. Comparison of the duration of hamstring flexibility improvement following termination of modified dynamic stretching, hold-relax, and static stretching. Korean Research Society of Physical Therapy. 2014;21(1):47-54.

8. Nelson RT. A comparison of the immediate effects of eccentric training vs static stretch on hamstring flexibility in high school and college athletes. N Am J Sports Phys Ther. 2006;1(2):56.

9. Puentedura EJ, Huijbregts PA, Celeste S, et al. Immediate effects of quantified hamstring stretching: Hold-relax proprioceptive neuromuscular facilitation versus static stretching. Phys Ther Sport. 2011;12(3):122-6.

10. Lim CG, The effects of proprioceptive neuromuscular facilitation (PNF) pattern exercise using the sprinter and the skater on balance and gait function in the stroke patients physical therapy. J Kor Soc Phys Ther. 2014;26(4):249-56.

11. Decoster LC, Scanlon RL, Horn KD, et al. Standing and supine hamstring stretching are equally effective. J Athl Train. 2004;39(4):330.

12. Cipriani D, Abel B, Pirrwitz D. A comparison of two stretching protocols on hip range of motion: Implications for total daily stretch duration. J Strength Cond Res. 2003;17(2):274-8.

13. Yuktasir B, Kaya F. Investigation into the long-term effects of static and PNF stretching exercises on range of motion and jump performance. J Bodyw Mov Ther. 2009;13(1):11-21.

14. Morishita K, Karasuno H, Yokoi Y, et al. Effects of therapeutic ultrasound on range of motion and stretch pain. J Phys Ther Sci. 2014; 26(5):711.

15. Solomon J, Shebshacvich V, Adler R, et al. The effects of TENS, heat, and cold on the pain thresholds induced by mechanical pressure in healthy volunteers. Neuromodulation: Technology at the Neural Interface. 2003;6(2):102-7.

16. Lee JW, Park AR, Hwang TY, Change in autonomic nerve responses after low-frequency transcutaneous electrical nerve stimulation. J Kor Soc Phys Ther. 2010;22(6):71-6. 
17. Vance CG, Dailey DL, Rakel BA, et al. Using TENS for pain control: the state of the evidence. Pain management. 2014;4(3):197-209.

18. De Weijer VC, Gorniak GC, Shamus E. The effect of static stretch and warm-up exercise on hamstring length over the course of 24 hours. J Orthop Sports Phys Ther. 2003;33(12):727-33.

19. DePino GM, Webright WG, Arnold BL. Duration of maintained hamstring flexibility after cessation of an acute static stretching protocol. J Athl Train. 2000;35(1):56.

20. Kolber MJ, Hanney WJ. The reliability and concurrent validity of shoulder mobility measurements using a digital inclinometer and goniometer: A technical report. Int J Sports Phys Ther. 2012;7(3):306.

21. Davis DS, Ashby PE, McCale KL, et al. The effectiveness of 3 stretching techniques on hamstring flexibility using consistent stretching parameters. J Strength Cond Res. 2005;19(1):27-32.

22. Maciel A, Câmara S. Influence of transcutaneous electrical nerve stimulation (TENS) associate with muscle stretching on flexibility gains. Braz J
Phys Ther. 2008;12(5):373-8.

23. Mannheimer JS. Electrode placements for transcutaneous electrical nerve stimulation. Phys Ther. 1978;58(12):1455-62.

24. Denegar CR, Perrin DH. Effect of transcutaneous electrical nerve stimulation, cold, and a combination treatment on pain, decreased range of motion, and strength loss associated with delayed onset muscle soreness. J Athl Train. 1992;27(3):200.

25. Chae YW, Kim SY, Kim JS, et al. The effects of tens and cold application on secondary thermal hyperalgesia in rats induced by muscle pain. J Kor Soc Phys Ther. 2004;16(2):181-94.

26. Sluka KA, Deacon M, Stibal A, et al. Spinal blockade of opioid receptors prevents the analgesia produced by tens in arthritic rats. J Pharmacol Exp Ther. 1999;289(2):840-6.

27. Jeong DH, Chang CH, Park RJ. A review of conception and developmental process of stretching in sports physical therapy. J Kor Soc Phys Ther. 2002;14(4):423-40. 